\title{
Cadre des indicateurs de surveillance de la maltraitance envers les enfants
}

\author{
Aimée Campeau, M.A.; Shazmeera Qadri, M.S.P.; Farah Barakat, B.A.; Gabriela Williams, M. Sc.; \\ Wendy Hovdestad, Ph. D.; Maaz Shahid, M. Sc. en santé publique; Tanya Lary, M.A.
}

Diffuser cet article sur Twitter

\begin{abstract}
Résumé
Le portefeuille de la santé du gouvernement du Canada effectue la surveillance de la violence envers les enfants en tant que problème de santé publique depuis les années 1990. L’Agence de la santé publique du Canada (ASPC) publie aujourd'hui le Cadre d'indicateurs de la maltraitance envers les enfants, qui prend place aux côtés d'autres cadres publiés par l'ASPC, comme le Cadre d'indicateurs de surveillance du suicide. Fondé sur un examen de la portée de revues de la littérature et de méta-analyses, ce cadre, avec l'outil interactif de données en ligne, présente des indicateurs de résultats et des facteurs de risque et de protection de la maltraitance envers les enfants, selon le sexe, l'âge et diverses variables sociodémographiques, aux échelles individuelle, familiale, communautaire et sociétale. Il va constituer une ressource précieuse en lien avec un problème qui affecte au moins un adulte canadien sur trois.
\end{abstract}

Mots-clés : maltraitance envers les enfants, violence physique, exposition à la violence conjugale, négligence, violence psychologique, abus sexuel

\section{Introduction}

La maltraitance envers les enfants est un problème de santé publique qui a des effets immédiats et à long terme sur la santé physique et mentale ${ }^{1}$. Les données de surveillance sont essentielles à la mise en œuvre de politiques et de programmes visant à prévenir et à contrer la maltraitance envers les enfants. L’Agence de la santé publique du Canada (ASPC) est responsable de la surveillance nationale de la maltraitance envers les enfants, que ce soit la violence physique, l'exposition à la violence entre partenaires intimes, la négligence, la maltraitance psychologique ou la violence sexuelle ${ }^{2}$. L'ASPC recueille actuellement des données sur la maltraitance envers les enfants à partir de données administratives provenant d'agences de protection de l'enfance, d'enquêtes auprès d'informateurs substituts et d'enquêtes par autodéclaration.
Le Cadre des indicateurs de surveillance de la maltraitance envers les enfants (CISME) réunit les données disponibles sur la maltraitance envers les enfants ainsi que sur les facteurs de risque et de protection aux échelles individuelle, familiale, communautaire et sociétale, afin d'appuyer la recherche et l'élaboration de politiques. Le CISME, publié pour la première fois dans ce numéro de Promotion de la santé et prévention des maladies chroniques au Canada, sert de complément à d'autres cadres d'indicateurs publiés par l'ASPC, à savoir des Indicateurs de surveillance du suicide (ISS), des Indicateurs des maladies chroniques au Canada (IMCC), du Cadre d'indicateurs de surveillance de la santé mentale positive (CISSMP) et du Cadre d'indicateurs de l'activité physique, du comportement sédentaire et du sommeil (APCSS).

\section{Méthodologie}

Le CISME a été élaboré à l'aide d'une méthode d'examen de la portée ${ }^{3}$. Les

\section{Points saillants}

- Le Cadre d'indicateurs de la maltraitance envers les enfants vient compléter d'autres cadres d'indicateurs publiés par l’Agence de la santé publique du Canada et présente des données sur les résultats et les facteurs de risque et de protection de la violence envers les enfants, aux échelles individuelle, familiale, communautaire et sociétale.

- Le tiers $(34,1 \%)$ de la population canadienne âgée de 15 ans et plus a été victime d'au moins un type de maltraitance durant l'enfance.

- La violence physique est le type de maltraitance le plus répandu $(27,4 \%)$, suivie de l'exposition à la violence entre partenaires intimes (10,6\%) et de l'abus sexuel $(8,1 \%)$.

- Des facteurs présents chez les parents tels que la maladie mentale, la consommation de substances et le fait d'avoir été victime de violence familiale peut exposer les enfants à un risque plus élevé de maltraitance.

recherches d'articles ont ciblé en priorité les revues systématiques, les méta-analyses et les analyses documentaires qui traitent des risques et des facteurs de protection liés à tout type de mauvais traitements infligés aux enfants. Les références figurant dans les articles consultés ont été examinées afin de trouver d'autres références. Les articles pertinents publiés en anglais entre 2004 et 2016 et divers rapports d'organismes de santé publique 
(comme l'Organisation mondiale de la santé) ont été consultés. La liste initiale d'environ 45 à 50 indicateurs a été raccourcie grâce à des consultations avec des experts en surveillance de la maltraitance envers les enfants ainsi qu'avec les ministères participant à l'Initiative de lutte contre la violence familiale, de manière à ce que la liste finale soit pertinente et puisse être mise en œuvre dans le cadre d'une approche en santé publique.

\section{Résultats}

Les données de l'Enquête sociale générale (ESG) de 2014 indiquent que plus du tiers $(34,1 \%)$ de la population âgée de 15 ans et plus a subi au moins un des types suivants de maltraitance durant l'enfance : violence physique ou sexuelle par un adulte ou exposition à la violence par les parents ou les tuteurs (tableau 1). Parmi les trois types de maltraitance subie durant l'enfance, la violence physique était la plus fréquente $(27,4 \%)$, suivie de l'exposition à la violence entre partenaires intimes $(10,6 \%)$ et de la violence sexuelle $(8,1 \%)$.

En raison du contexte familial dans lequel la majorité de la maltraitance infligée aux enfants se produit, les facteurs de risque et de protection sont axés sur les caractéristiques parentales ou familiales plutôt que sur les caractéristiques des enfants. Des facteurs comme la maladie mentale des parents, la toxicomanie et l'expérience antérieure de violence familiale peuvent exposer les enfants à un risque supérieur de maltraitance.

\section{Conclusion}

Les expériences vécues durant l'enfance sont un important déterminant social de la santé ${ }^{4}$. Le CISME fournit des données probantes sur les contextes et les facteurs de risque et de protection pour aider à cibler les stratégies de prévention et de lutte contre la maltraitance envers les enfants.

Le CISME est un document évolutif qui sera révisé périodiquement et mis à jour à mesure que de nouvelles données seront disponibles. Un outil interactif en ligne sur l'Infobase de la santé publique du gouvernement du Canada fournira une ventilation des indicateurs selon le sexe, l'âge, la province ou le territoire et le statut vis à vis de l'immigration.

Les lacunes en matière de données indiquées dans ce cadre, particulièrement à l'échelle communautaire et sociétale, vont être comblées grâce à l'élaboration en cours de données, au moyen d'enquêtes et de données administratives.

\section{Conflits d'intérêts}

Les auteurs déclarent n'avoir aucun conflit d'intérêts.

\section{Contributions des auteurs et avis}

AC, SQ, GW, MS et WH ont contribué à la conception et la conceptualisation du travail, ainsi qu'à l'acquisition, l'analyse et l'interprétation des données. FB, WH et TL ont contribué à l'interprétation des données et à la rédaction et la révision du manuscrit.

Le contenu de l'article et les points de vue qui y sont exprimés n'engagent que les auteurs et ils ne correspondent pas nécessairement à ceux du gouvernement du Canada.

\section{Références}

1. Organisation mondiale de la santé. La maltraitance des enfants : principaux faits [Internet]. Genève (Suisse) : Organisation mondiale de la santé; 2016 [consultation le 10 septembre 2019]. En ligne à : https://www.who .int/fr/news-room/fact-sheets/detail /child-maltreatment

2. Agence de la santé publique du Canada. Les mauvais traitements infligés aux enfants au Canada [Internet]. Ottawa (Ontario) : Agence de la santé publique du Canada; 2012 [modification le 26 juillet 2012; consultation le 10 septembre 2019]. En ligne à : https:// www.canada.ca/fr/sante-publique /services/promotion-sante/arretons -violence-familiale/ressources-prevention /enfants/mauvais-traitements-infliges -enfants-canada.html

3. Ganann R, Ciliska D, Thomas H, Expediting systematic reviews: methods and implications of rapid reviews. Implement Sci. 2010;5:56-66. doi:10.1186 /1748-5908-5-56.

4. Agence de la santé publique du Canada. Déterminants sociaux de la santé et inégalités en santé [Internet]. Ottawa (Ontario) : Gouvernement du Canada; 2018 [modification le 28 juin 2019; consultation le 10 septembre 2019]. En ligne à : https://www.canada.ca /fr/sante-publique/services/promotion -sante/sante-population/est-determine -sante.html
5. Statistique Canada. La violence familiale au Canada : un profil statistique, 2015. Ottawa (Ontario) : Statistique Canada; [modification le 4 mai 2017; consultation le 10 septembre 2019]. [Statistique Canada, $\mathrm{n}^{\circ}$ au catalogue : 85-002-X].

6. Walsh CA, MacMillan HL, Trocmé N, Jamieson E, Boyle MH. Measurement of victimization in adolescents: development and validation of the Childhood Experiences of Violence Questionnaire. Child Abuse Negl. 2008;32(11):1037-1057. 


\section{TABLEAU 1}

\section{CADRE DES INDICATEURS DE SURVEILLANCE DE LA MALTRAITANCE ENVERS LES ENFANTS STATISTIQUES RAPIDES, CANADA, ÉDITION 2019}

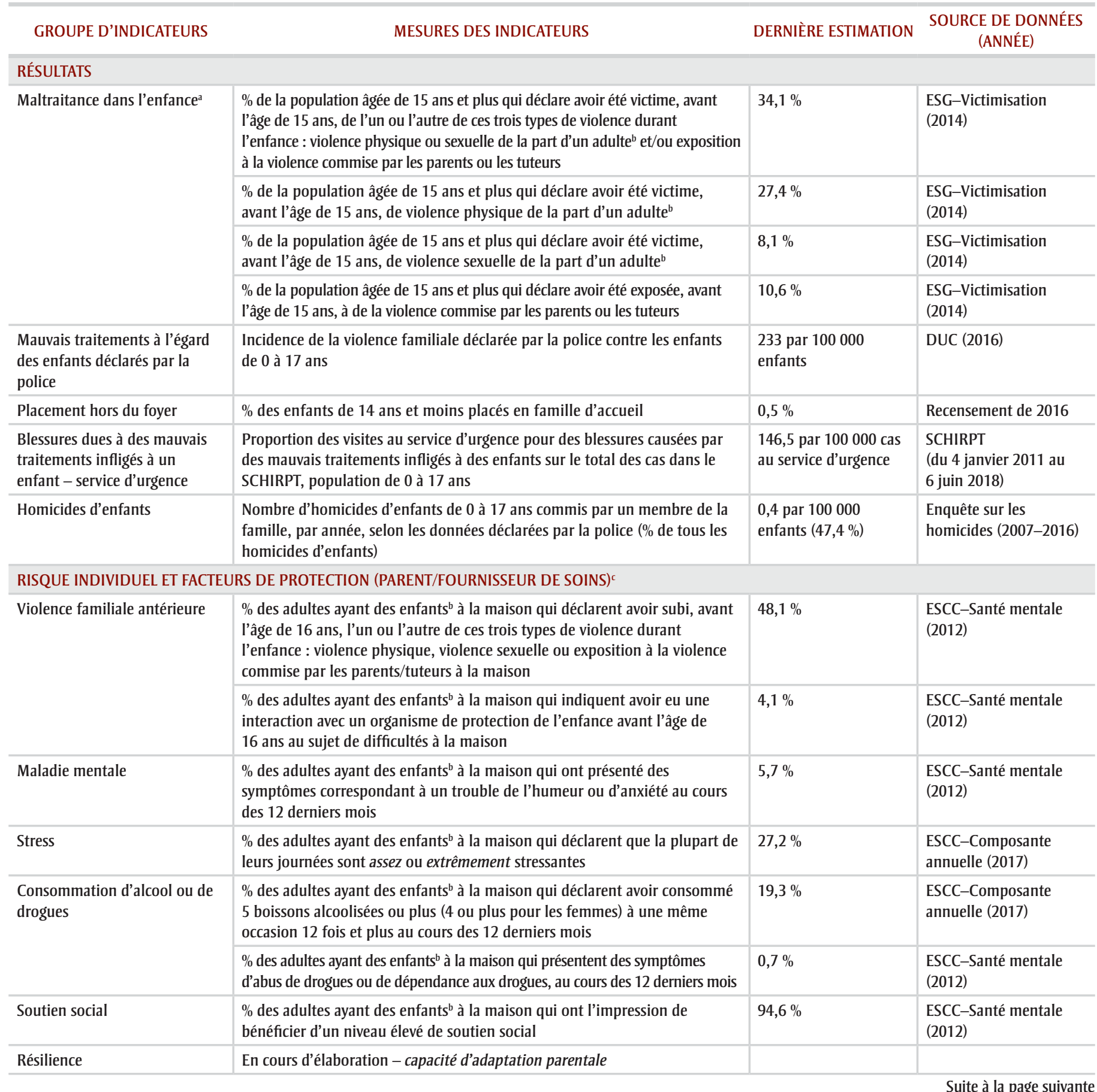


Relations familiales

Stabilité de la

dynamique familiale

Éducation des enfants soins

\% des parents/tuteurs d'enfants âgés de 1 à 9 ans qui font état de caractéristiques associées à un fonctionnement familial de niveau élevé $\%$ des élèves de la $6^{\mathrm{e}}$ à la $10^{\mathrm{e}}$ année qui sont d'accord ou tout à fait d'accord avec l'énoncé selon lequel ils obtiennent l'aide et le soutien émotionnels dont ils ont besoin dans leur famille

$\%$ des élèves de la $6^{\mathrm{e}}$ à la $10^{\mathrm{e}}$ année qui sont d'accord ou tout à fait d'accord avec l'énoncé selon lequel ils se disputent souvent avec leurs parents

\% des enfants de 1 à 9 ans qui sont parfois ou souvent exposés à des adultes ou à des adolescents qui se battent, qui frappent ou essaient de blesser d'autres personnes à leur domicile, selon la déclaration du parent ou du tuteur ${ }^{d}$

En cours d'élaboration - présence d'un fournisseur de soins temporaire non biologique à la maison (p. ex. partenaire du parent)

$\%$ des enfants de 1 à 9 ans qui subissent parfois, souvent ou toujours un châtiment physique comme forme de discipline, selon la déclaration du parent ou du tuteur $^{\mathrm{d}}$

En cours d'élaboration - faible sensibilisation du fournisseur de soins aux besoins des enfants ou aux stades de développement des enfants

$\%$ des adultes ayant des enfants ${ }^{\mathrm{b}}$ à la maison qui déclarent que les soins à des membres de la famille sont le principal facteur de leur stress quotidien

En cours d'élaboration - besoins spéciaux d'un enfant qui peuvent accroître le fardeau du fournisseur de soins de l'enfant

Grossesse précoce

Revenu du ménage \% des premières naissances vivantes chez les mères de moins de 20 ans

$\%$ de la population âgée de moins de 18 ans dans des familles économiques vivant sous le seuil de faible revenu, après impôts

(ANNÉE)

EJC-PMR

(2010-2011)

\begin{tabular}{|l|l|}
\hline $91,1 \%$ & $\begin{array}{l}\text { EJC-PMR } \\
(2010-2011)\end{array}$ \\
\hline $64,8 \%$ & HBSC (2013-2014) \\
\hline $21,7 \%$ & HBSC (2013-2014) \\
\hline $2,6 \%$ & $\begin{array}{l}\text { EJC-Enfant } \\
(2010-2011)\end{array}$ \\
\hline $0,5 \%$ & $\begin{array}{l}\text { EJC-Enfant } \\
(2010-2011)\end{array}$ \\
\hline
\end{tabular}

(2010-2011)

\begin{tabular}{|l|l|}
\hline $18,1 \%$ & $\begin{array}{l}\text { ESCC-Composante } \\
\text { annuelle (2016) }\end{array}$ \\
\hline
\end{tabular}

RISQUES COMMUNAUTAIRES ET FACTEURS DE PROTECTION

Disponibilité et accessibilité des services

Environnement social
En cours d'élaboration - services communautaires pour les parents et les familles

$\%$ des parents/tuteurs d'enfants de 1 à 9 ans qui font partie d'organismes bénévoles (groupes scolaires ou religieux, associations communautaires ou ethniques)

En cours d'élaboration - instabilité résidentielle ou appauvrissement du quartier

En cours d'élaboration - proximité des points de vente d'alcool

Environnement physique

\section{RISQUES SOCIÉTAUX ET FACTEURS DE PROTECTION}

Politique publique favorisant la En cours d'élaboration - systèmes de soutien officiels pour les familles santé

Normes culturelles et sociales

En cours d'élaboration - croyances sociales/culturelles concernant les châtiments physiques comme moyen disciplinaire pour élever et éduquer les enfants

Inégalités

En cours d'élaboration - droits des enfants

Abréviations : DUC, Déclaration uniforme de la criminalité; ECR, Enquête canadienne sur le revenu; EJC-Enfant, Enquête sur les jeunes canadiens - questionnaire de l'enfant; EJC-PMR, Enquête sur les jeunes canadiens - questionnaire de la personne la mieux renseignée; ESCC-Composante annuelle, Enquête sur la santé dans les collectivités canadiennes - Composante annuelle; ESCC-Santé mentale, Enquête sur la santé dans les collectivités canadiennes - Santé mentale; ESG, Enquête sociale générale; HBSC, Comportements de santé des jeunes d'âge scolaire; SCHIRPT, Système canadien hospitalier d'information et de recherche en prévention des traumatismes; SEC-BDN, Statistiques de l'état civil - Base de données sur les naissances (sauf le Québec).

Remarque : «En cours d'élaboration » désigne les mesures pour lesquelles la source de données n'est pas disponible à l'heure actuelle ou pour lesquelles d'autres recherches sont nécessaires pour déterminer une mesure prometteuse et une source de données.

${ }^{a}$ Les estimations de ce cadre d'indicateurs ont été calculées d'une manière conforme à celles du rapport de Statistique Canada intitulé La violence familiale au Canada : un profil statistique, $2015^{5}$, à l'aide d'une version légèrement différente de la base de données. Toutes les réponses positives à toutes les questions sur la maltraitance durant l'enfance ont été comptées comme des signes de maltraitance. Par contre, les règles d'analyse originales du Questionnaire sur les violences subies dans l'enfance (CEVQ) ${ }^{6}$ prennent en compte toutes les réponses positives, sauf pour deux sous-types de violence physique (les moins graves) et l'exposition à la violence entre partenaires intimes si celle-ci s'est produite moins de trois fois.

${ }^{b}$ Un adulte est défini comme une personne de 18 ans et plus; un enfant est défini comme une personne de moins de 18 ans.

' Les caractéristiques parentales, et non les caractéristiques des enfants, ont été considérées comme des facteurs de risque ou de protection pour la maltraitance des enfants. Cette approche repose sur une perspective de santé publique selon laquelle les facteurs de risque parentaux peuvent représenter les résultats potentiels de la maltraitance des enfants (comme la toxicomanie prénatale ou la violence)

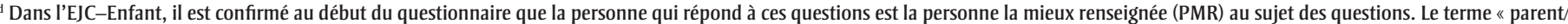
tuteur » est utilisé ici pour simplifier. 